\title{
The generalized pseudophase model: Treatment of multiple equilibria in micellar solutions
}

\author{
Claudio MINERO and Ezio PELIZZETTI \\ Dipartimento di Chimica Analitica - Università di Torino \\ via P.Giuria 5, 10125 Torino, Italy
}

\begin{abstract}
A review of the generalised pseudophase model is presented emphasising the theoretical premises and the hypotheses on which it is based. The calculations of $\mathrm{cmc}$ and the degree of dissociation of surfactants is presented for simple cases as well as for mixture of surfactants and for surfactants subject to multiple chemical equilibria.
\end{abstract}

\section{INTRODUCTION}

Many thermodynamic theories of micellization based on or leading to the mass action law (ref.1-5) and on quantum statistical mechanical formalism (ref.6,7) can be used to calculate the $\mathrm{cmc}$, the aggregation number and in some cases the counterion binding. For their nature, they assume the molecular arrangement of micellar constituents or, at least, they impose some constraints on the position of head groups. Recently, within the SCFA theory (ref.8) the localisation or the surface density of head groups was calculated from the equilibrium properties of the formed structure. The theory is also able to accommodate the difference among linear and branched chains and is based on a lattice model. This implies the calculation of the volume fraction of tail segments for different micelle shape functions through some type of chain statistics. Although the theory is quite advanced and sophisticated, the application to experiments in micellar solutions is faraway and a big computational burden can be expected.

Simple thermodynamic theories and hypotheses give less refined treatments but can be applied with minor efforts to experimental data and in some cases lead to analytical results. A well known simple theory is the pseudophase approximation. A number of different kind of experiments in micellar solutions were evaluated using this approach. The pseudophase model was successful in explaining binary ionic (ref.8) and binary non ionic (ref.9) surfactant mixtures, as well as the effect of different counterions (ref.10) and multicomponent systems (ref.11). Often the counterion binding was overpassed by assuming it constant or assuming related quantities as constant parameters. Also electrostatic approaches were developed (ref.12) to account for micelle and medium variations.

Some refinements to this approximation were suggested in the pseudophase generalised thermodynamic approach (ref.13), in which hydrophobic and electrostatic contributions to transfer coefficients were explicit . These improvements have been proved 
quite successful in treating micellar kinetic in the presence of different counterions (ref.14) and mixed surfactants (ref.15). The model also foresees correctly the acid-base equilibria shifts (ref.16) in micellar solutions. The power of the model comes out from the possibility of applying the same formalism to different kind of experiments and from the possibility of comparing or measuring the parameters of the model with independent techniques. The model is based on the approximation that the solution can be modelled with two pseudophases, as in the older pseudophase models, and on the assumption of the "dressed" micelle, by means of which all bound species are considered part of the aggregate and do not contribute to the bulk ionic strength. An interface, where the polar or charged heads of the amphiphilic molecules are located, is presumed at some distance from the center of the micelle. The aggregation number was assumed known or independent of medium variations.

This paper considers the framework of the generalised pseudophase model, its previsions about the degree of dissociation and the critical micelle concentration and the case of systems subject to chemical equilibria.

\section{BASIC THEORY}

The equilibrium condition applied to the formation of a micelle composed of surfactant, condensed counterions and coions and several other solubilized species implies that

$$
\mu_{\mathrm{MIC}}=\sum v_{\mathrm{i}} \mu_{\mathrm{i}}^{\mathrm{f}}
$$

where $v_{i}$ is the stoichiometric coefficient of the species $i$ in the equilibrium micelle and the superscript $f$ refers to components in solution (free). When considering the surfactant (subscript $\mathrm{m}$ ) $v_{\mathrm{m}}$ is the aggregation number $\mathrm{N}$. By assuming that the internal chemical potential of the micelle is the sum of the chemical potentials of the components in the micelle (superscript b), i.e. $\mu^{\circ}{ }_{M 1 C}=\sum v_{i} \mu_{i}^{b}$, and taking the usual definition of the electrochemical potential $\mu_{\mathrm{i}}$, thus it follows

$$
\sum_{i} v_{i}\left(\mu_{i}^{f}-\mu_{i}^{b}\right)+=R T \ln \frac{\varphi}{(1-\varphi)}+R T \ln \gamma_{M J c}
$$

and the mass action law

$$
\sum_{i} v_{i} \ln \left(K_{i}^{0} K_{i}^{e}\right)+\sum_{i} v_{i} \ln \frac{\left[I_{b}\right]}{\left[I_{f}\right]} \frac{\varphi f_{i}}{(1-\varphi)}=\ln \frac{\varphi}{(1-\varphi)}+\ln \gamma_{M I C}
$$

where $\varphi$ is the volume fraction of micelles, $\left[\mathrm{I}_{b}\right]$ and $\left[\mathrm{I}_{\mathrm{f}}\right]$ are the molar concentrations of bound and free species over the total volume of the solution, $\gamma_{M C}$ is the activity coefficient of the micelle and $f_{i}$ is the fraction of micellar volume that the species $i$ is allowed to occupy. The stoichiometric coefficients $v_{i}$ can be easily expressed as a function of $\varphi$ or the concentration of micellized surfactant $C_{d}: V_{i}=\left[I_{b}\right] N / C_{d}=\left[I_{b}\right] V_{\text {mid }} / \varphi$, where $V_{\text {mic }}$ is the molar volume of the micelle.

The term RTln $\left(\mathrm{K}_{\mathrm{i}}{ }^{\mathrm{O}}\right)=\left(\mu_{\mathrm{i}}{ }^{\mathrm{of}}-\mu_{\mathrm{i}}{ }^{\mathrm{ob}}\right)$ contains all the differences in the coupling work in the two environments that are not strictly coulombic ( dipole-dipole, dipole-induced dipole, higher order electrostatic interactions, specific interactions with the media, solvation). 
Statistical thermodynamic approach definition of $\mu_{i}{ }^{\circ}$ is also possible (ref.17) in terms of the internal partition function (rotational, vibrational, electronic etc.), of the momentum partition function, and of the non electrostatic coupling work of the species against the medium. In this regard, the equilibrium constant is solely determined by the configuration integral ( depending on the interaction potential) and kinetic energy terms have no influence on the equilibrium properties (ref.18). In other words, in the equilibrium constant the terms of kinetic energy and "internal energy" cancel. In the pseudophase approximation $\mathrm{K}_{\mathrm{i}}{ }^{\circ}$ is usually considered constant with respect to medium variations although, assuming $\mu_{b}^{n}$ constant is not always a good hypothesis, because short range contributions can depend on the equilibrium composition of the micelle. For example, an optimum $\mathrm{N}_{\text {aggr }}$ implies that $\mu^{\circ}{ }_{i}$ (and $K_{i}{ }^{\circ}$ ) goes through a maximum at $\mathrm{N}_{\text {aggr }}$ or decreases as $\mathrm{N}_{\text {aggr }}$ increases (ref.19).

The electrostatic partition term $\ln \left(\mathrm{K}_{i} \mathrm{e}\right)=\ln \left(\gamma_{i}^{\mathrm{f}} / \gamma_{\mathrm{i}}^{\mathrm{b}}\right)$, where $\gamma_{i}$ are the activity coefficients of the species $i$, is a mean field approximation which relates the difference of the electrostatic coupling works of ions with the two media (surface of the micelle or bulk solution at a given ionic strength). The contribution of the coupling work with the bulk was often neglected. Thus:

$$
-\ln K_{j}{ }_{j}=\frac{z_{i} e \psi_{s}}{K_{B} T}+\frac{z_{i}^{2} B_{l} \kappa}{2\left(1+\kappa r_{d i}\right)}
$$

where for the activity coefficient of the bulk ions $\gamma_{i}^{f}$ the linearized Poisson Boltzmann equation is used, $\mathrm{B}_{\mathrm{l}}=\mathrm{e}^{2} / 4 \varepsilon \varepsilon_{0} \mathrm{~K}_{\mathrm{B}} \mathrm{T}=7.192 \AA$ (at $25^{\circ} \mathrm{C}$ ), $\mathrm{r}_{\mathrm{di}}$ is the mean approach distance of the ion with other ions in solution and $z_{i}$ the ion charge. Seeing that $r_{d i}$ depends on the type of counterions, a value between 3.8 and $4.25 \AA$ would be appropriate for most experimental conditions (ref.15).

We can calculate $\psi$ and $\kappa$ by means of some model of the micelle. Since micelles and bound counterions do not contribute to the ionic strength, the Debye length $\kappa(\kappa=0.329 \sqrt{ } \mathrm{I}$ at $25^{\circ} \mathrm{C}$ ) can be calculated from

$$
I=1 / 2 \sum_{i} z_{i}^{2}\left[I_{f}\right]
$$

The surface potential depends on the amount of "condensed" ions and, in the case of spherical symmetry, it can be related to the net surface charge through the PoissonBoltzmann equation (solved numerically) or known analytical formulas. In the DebyeHückel approximation eq.(6) holds:

$$
\psi_{s}=\frac{Q B_{1} K_{\mathrm{B}} T}{e R_{\text {mic }}\left(1+\kappa R_{\text {mic }}\right)}
$$

where $Q$ is the equilibrium net surface charge and $R_{m i c}$ is the radius of the micelle. The possible positions of the ions at the micellar surface have been discussed in details (ref.20). Although measurements by neutron scattering revealed that the head group region in micelles is thicker than the thickness of a single head group (ref.21), by considering the model of the dressed micelle and assuming that all counterions are placed at the plane of surfactant head groups, the potential $\psi$ sensed by bound ions is the surface potential of the micelle originated by $Q$ smeared net surface charges.

$$
Q=z_{m} N+V_{m i c} \sum_{i} z_{i}\left[I_{b}\right] / \varphi
$$


Both $Q$ and I necessary to calculate $K^{e_{i}}$ can be expressed as a function of concentrations $\left[I_{b}\right]$ and $\left[I_{f}\right]$ if eq. (2) can be solved for equilibrium concentrations of the species in micelle and in bulk. A particular solution that satisfies both the mass action law eq.(2) and the pseudophase approximation (see below) is the system of equations obtained by separately equating to zero the terms of the species $i$

$$
\ln \left(K_{i}{ }^{n} K_{i}{ }^{e} f_{j}\right)+\ln \frac{\left[I_{f}\right]}{\left[I_{b}\right]} \frac{\varphi}{(1-\varphi)}=0
$$

except for a reference species ( for example the surfactant) for which

$$
\ln \left(K_{m}{ }^{n} K_{m}{ }^{e} f_{m}\right)+\ln \frac{\left[m_{f}\right]}{\left[C_{d}\right]}-\frac{N-1}{N} \ln \frac{\varphi}{(1-\varphi)}-\frac{1}{N} \ln \gamma_{M I C}=0
$$

Eq. (8a) is the pseudophase relationship obtained from the usual assumption $\left(\mu_{j}{ }^{f}=\mu_{i}^{b}\right)$ that the equilibrium exists between two pseudophases (micelle and bulk). From the mass balance $C_{j}=\left[I_{b}\right]+\left[I_{f}\right]$ the micellar and bulk concentrations for all the species can be calculated as a function of hydrophobic transfer coefficients (knows or parameters of the model), electrostatic transfer coefficients ( calculated through eqs.6-7), the volume fraction $\varphi$ of micelles and the aggregation number

$$
\begin{array}{ll}
{\left[I_{b}\right]=\frac{C_{i} K_{i}{ }^{0} K_{i}^{e} f_{i} \varphi}{\left(1+\varphi\left(K_{i}{ }^{0} K_{i}^{e} f_{i}-1\right)\right)}} & {\left[I_{f}\right]=\frac{C_{i}(1-\varphi)}{\left(1+\varphi\left(K_{i}^{0} K_{j}^{e} f_{j}-1\right)\right)}} \\
C_{d}=\frac{C_{m} K_{m}{ }^{n} K_{m}{ }^{e} f_{m}\left(\gamma_{M I C}\right)^{-1 / N} A}{1+K_{m}{ }^{n} K_{m}{ }^{e} f_{m}\left(\gamma_{M I C}\right)^{-1 / N} A} & {\left[m_{f}\right]=\frac{C_{m} A}{\left(1+K_{m}{ }^{0} K_{m}{ }^{e} f_{m}\left(\gamma_{M I C}\right)^{-1 / N} A\right)}}
\end{array}
$$

where $A=\left(\varphi /(1-\varphi)^{((\mathrm{N}-1) / \mathrm{N})}\right.$.

By comparing eq.(9b) with eq.(9a) we see that the pseudophase approximation applied to the surfactant holds when $\mathrm{N} \rightarrow \infty$. Depending on $\gamma_{M I C}$ and $\varphi$, the approximate formula (9a) can be reliable (with negligible error) when $N \geq 50$, i.e. when the micelle aggregation number is greater than 50 , the mass action model becomes equivalent to the pseudophase separation approach as previously suggested (ref.22).

The system of equations (5,7), with the aid of eq.(9), completely defines the degree of dissociation and the bulk ionic strength in the presence of micelles of given aggregation number and of given radius, and the bound and free concentrations of the species as function of the volume fraction of micelles, the analytical concentration $\mathrm{C}_{\mathrm{i}}$ of the species and, in addition to geometric constants, of only one parameter per species, namely the hydrophobic transfer constant $\mathrm{K}^{\mathrm{n}}$. Several attempts to include specific interactions of the ions with the micelle by means of " $a$ priori" assumed Volmer isotherm are reported (ref.23) in the framework of the non-linearized Poisson Boltzmann electrostatic treatments. The present model avoid this "a priori" assumption and is equally applicable to ionic and nonionic species as well as to the surfactant itself. 


\section{CRITICAL MICELLE CONCENTRATION}

Since eq. (9b) refers to the surfactant, in the simplest case the cmc can be obtained in the limit $\varphi<1$ :

$$
\mathrm{cmc}=\frac{1}{\mathrm{v}_{\mathrm{m}} \mathrm{K}_{\mathrm{m}}^{\mathrm{o}} \mathrm{K}_{\mathrm{m}}^{\mathrm{e}}}
$$

where $\mathrm{K}_{\text {:11 }}^{-}$refers to the micelle in equilibrium at the cmc and the approximation $\varphi=v_{m} C_{d}$ was used. The cmc can be calculated numerically from the internal consistency of eq. $(5,7)$ with eq. $(9 \mathrm{~b})$ at given salt and total surfactant concentrations in the limit $\varphi \ll 1$.

For non-ionic surfactants $\mathrm{K}_{\mathrm{m}}^{\mathrm{e}}=1$ and the predicted cmc is constant as the total concentration of the surfactant is varied. So called salting-out effects, if present, can be accounted for by evaluating the activity coefficient of the monomer (its coupling work) in the bulk solution with extended formulas like that of Davies. In this case, $\mathrm{K}_{\mathrm{m}}{ }_{\mathrm{m}}$ will depend on the ionic strength even when the surfactant does not have a net charge.

In the case of ionic surfactants, the monomer has the same charge of the micelle: $\mathrm{Ke}_{\mathrm{m}}$ will be lower than unity and will increase ( towards unity) with the added salt concentration, while the $\mathrm{cmc}$ will be depressed till the limit $1 / \mathrm{v}_{\mathrm{m}} \mathrm{K}_{\mathrm{n}}{ }_{\mathrm{m}}$.

Solution of the system $(5,7)-(9)$ is possible only via numerical algorithms which take particular care in assuring convergence. The model presents some advantages compared to other similar models because only two non-linear equations in two unknown have to be simultaneously solved. Some examples of numerical solutions, obtained using the OHW equation (ref.24) for the charge-potential relationship and by considering for simplicity a system composed of the surfactant and an added salt with a common counterion, are reported in Figs.1-2 for different values of parameters.
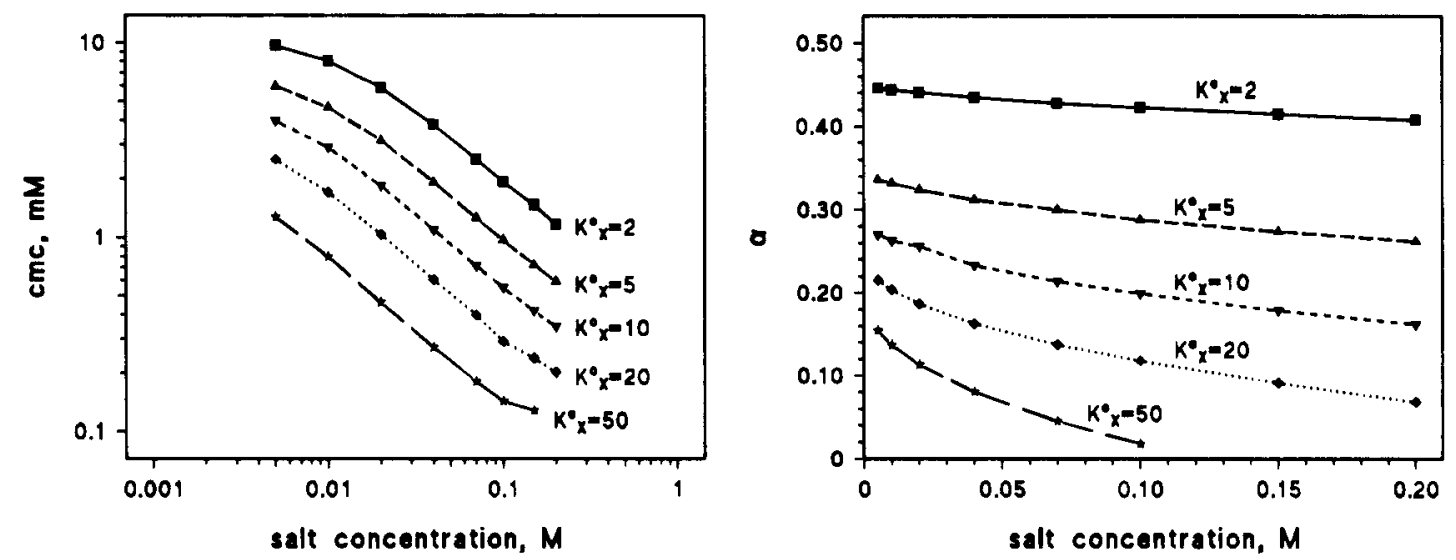

Fig.1. Calculated values of $\mathrm{cmc}$ and degree of dissociation $\alpha$ according to eq.(10) and (7) for a ionic surfactant SX as a function of concentration of an excess salt MX at different values of the hydrophobic transfer constant of the counterion $X$. Radii of all ions $2.5 \AA, \mathrm{K}_{\mathrm{m}}^{\mathrm{n}}=20000, \mathrm{~K}_{\mathrm{M}}^{\mathrm{n}}=5, \mathrm{~N}_{\mathrm{aggr}}=100$, micelle radius $25 \AA$.

Fig. 1 shows the effect on the cmc of an increasing concentration of salt. The hydrophobic transfer constants of the surfactant corresponds to free energy of transfer of about $5.8 \mathrm{Kcal} \mathrm{mole}^{-1}$ and is consistent with the estimated values of transfer per $-\mathrm{CH}_{2}$ group (450 cal/ $\mathrm{CH}_{2} \times 12-\mathrm{CH}_{2}$ - groups). The calculated values of $\mathrm{cmc}$ and $\alpha$ lie in the correct experimental range. The hydrophobicity of the counterion affects markedly the $\mathrm{cmc}$ value. There is a difference of an order of magnitude in the cmc passing from a hydrophilic counterion to a hydrophobic one. The $\mathrm{K}^{\mathrm{n}} \mathrm{X}$ values reported in Fig. 1 correspond to absolute 
free energy of transfer from water to micelle around $0.4-2.3 \mathrm{kcal} \mathrm{mole}^{-1}$ and can be estimated independently through theoretical models and methods proposed in literature (ref.6-8) taking into account all factors other than coulombic ones.

It is noteworthy that a linear relationship between $\log (\mathrm{cmc})$ and $\log ($ [added salt]) is obtained at fixed aggregation number, although a quick inspection of eq.(10) does not offer any particular evidence. Best fit straight lines to the calculated points show good correlation coefficients when the added salt is at least ten times the $\mathrm{cmc}$. From the slope of these straight lines, simpler theories (ref.25) deduce the degree of dissociation. In general, it is merely a coincidence that the calculated degree of dissociation (from eq. (7), $\alpha=$ $\mathrm{Q} / \mathrm{z}_{\mathrm{m}} \mathrm{N}_{\mathrm{aggr}}$ ) and that calculated from the experimental salt dependence of the cmc agree. These values can be compared with $\alpha$ reported in Fig.1, right, and rarely agree with those found with other experimental techniques (ref.19,26).

Fig. 1 shows that the effect of salt, although depending on the absolute value of $\mathrm{K}^{\mathrm{n}} \mathrm{X}$, is not outstanding except at very high concentrations where the degree of dissociation tends to zero. By adding the solution other chemical species with charges and/or hydrophobic transfer constants bigger than those here used, the computed degree of dissociation is not changed if their concentration is lower than $1 \times 10^{-4} \mathrm{M}$. This fact is important when extraneous molecular species are added to the surfactant system in order to probe the micellar environment (i.e. in kinetic experiments).
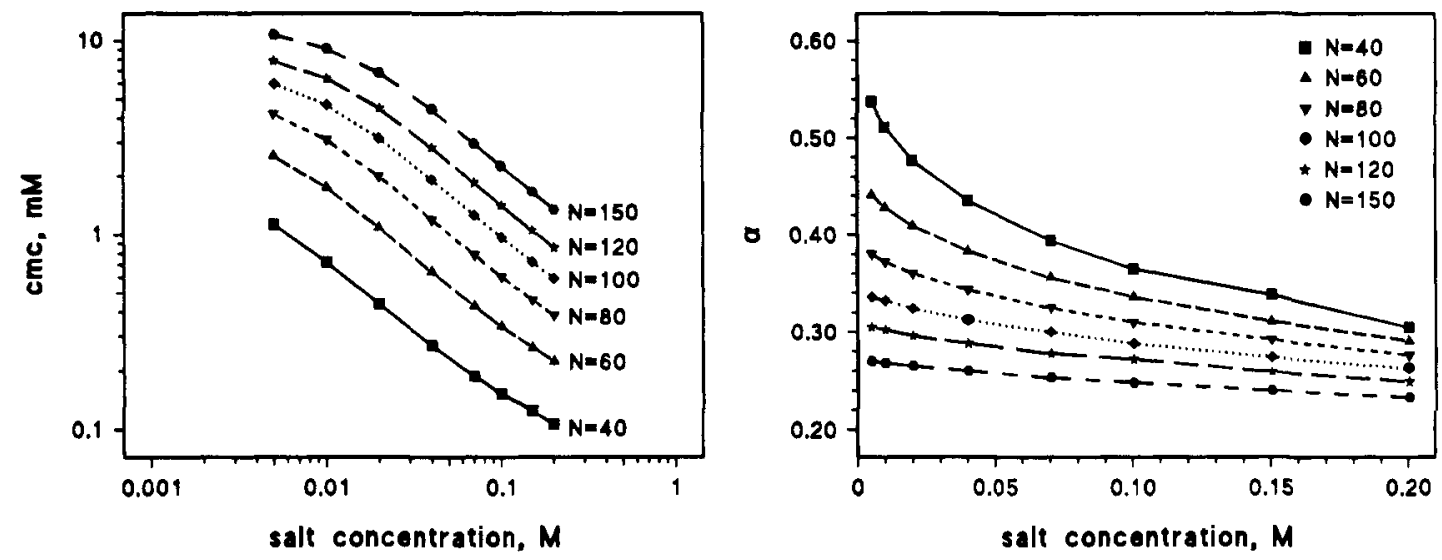

Fig.2. Calculated values of $\mathrm{cmc}$ and degree of dissociation $\alpha$ according to eq. (10) and (7) for a ionic surfactant SX as a function of concentration of an excess salt $\mathrm{MX}$ at different values of the aggregation number $\mathrm{N}$ (or surfactant molar volume $\mathrm{v}_{\mathrm{m}}$ ). Radii of all ions $2.5 \AA, \mathrm{K}_{\mathrm{m}}^{\circ}=20000, \mathrm{~K}^{\circ}{ }_{\mathrm{M}}=\mathrm{K}^{\circ} \mathrm{X}=5$, micelle radius $25 \AA$.

Fig.2 shows the effect on the cmc of the aggregation number or of the molar volume of the surfactant at fixed micellar radius. Surfactants with higher molar volumes ( i.e. branched tails or bigger heads) have lower cmc's. As $\mathrm{N}_{\text {aggr }}$ grows $\alpha$ decreases. It can be noted that at values of $\mathrm{N}_{\mathrm{aggr}}$ usually measured in surfactant systems $\left(70 \leq \mathrm{N}_{\text {aggr }} \leq 120\right)$ the degree of dissociation at the cmc does not change significantly and that the calculated behaviour agrees with the fact that at $\mathrm{N}_{\text {aggr }} \rightarrow 1$ the surfactant will become fully dissociated.

Arbitrary mixtures of salts can be easily taken into account. In the case of $2: 1$ electrolytes or of more complex mixtures, the appropriate relationship between net surface charge and potential must be used in place of eq.(6).

The surfactant concentration does not change $\alpha$ significantly, except when the concentration of added salt is very low, while the salt concentration has a strong effect (Fig.1,right) mainly when the hydrophobic transfer constant of the counterion is high. This effect is of special relevance mainly in studies of micellar growth (ref.27). The model is 
therefore able to take into account both experimental observations (the salt effect) and accepted literature hypotheses about the constancy of the degree of dissociation over large regions of surfactant concentration. This last hypothesis is commonly used in the extrapolation of experimental data to zero surfactant concentration (e.g. in light scattering experiments (ref.26)).

In all the calculations presented above, the micelle aggregation number was constant and $\mathrm{K}_{\mathrm{m}}^{\mathrm{m}}$ was taken as the non-coulombic transfer constant for the most probable monodisperse micelle, whereas at high salt concentration the constancy of $\mathrm{N}_{\text {aggr }}$ is no more reasonable. When also the aggregation number has to be evaluated, a structural model of the micelle is needed and other more refined techniques are desirable: they have to take into account the variation of $\mathrm{K}_{\mathrm{m}}^{\mathrm{o}}$ as function of the aggregation number and of the actual micellar geometry, together with a statistical thermodynamic analysis of the whole solution. Yet, at moderate salt concentrations or for systems not subject to a very saltdependent grow, the model is encouraging. Since at high salt concentrations it is known that micelles grow and can change shape, the actual behaviour of $\alpha$ can be described changing the formula (6) when a transition from spherical to cylindrical shape is conceivable, using for the geometry concerned the proper relationship between net surface charge and potential.

The $\mathrm{cmc}$ of a mixture of two surfactant of molar volumes $\mathrm{v}_{1}$ and $\mathrm{v}_{2}$ at total concentrations $\mathrm{C}_{\mathrm{m} 1}$ and $\mathrm{C}_{\mathrm{m} 2}$ is given by:

$$
\mathrm{cmc}=\frac{\mathrm{K}_{\mathrm{m} 2}^{\mathrm{o}} \mathrm{K}_{\mathrm{m} 2}+\mathrm{S} \mathrm{K}_{\mathrm{m} 1}^{\mathrm{o}} \mathrm{K}_{\mathrm{m} 1}}{\left(\mathrm{v}_{1}+\mathrm{v}_{2} \mathrm{~S}\right) \mathrm{K}_{\mathrm{m} 1}^{\mathrm{o}} \mathrm{K}_{\mathrm{m} 1}^{\mathrm{e}} \mathrm{K}_{\mathrm{m} 2}^{\mathrm{o}} \mathrm{K}_{\mathrm{m} 2}^{\mathrm{e}}}
$$

where $\mathrm{cmc}$ is the sum of the individual cmc's and $\mathrm{S}$ is the ratio of the concentrations of the two surfactants in the equilibrium micelle. The ratio $S$ is easily evaluated from the solution of the quadratic equation $\mathrm{S}^{2} \mathrm{v}_{2}\left(\mathrm{~K}_{1}-\mathrm{K}_{2}\right)+\mathrm{S}\left(\left(\mathrm{K}_{1}-\mathrm{K}_{2}\right) \mathrm{v}_{1}+\mathrm{C}_{\mathrm{m} 1} \mathrm{~K}_{1} \mathrm{~K}_{2}\right)-\mathrm{C}_{\mathrm{m} 2} \mathrm{~K}_{1} \mathrm{~K}_{2}=0$, where $\mathrm{K}_{\mathrm{i}}=\mathrm{K}_{\mathrm{mi}}^{\mathrm{n}} \mathrm{K}_{\mathrm{mi}}$. The case $\mathrm{v}_{1}=\mathrm{v}_{2}$ was previously reported (ref.15). In the case that $\mathrm{K}_{1}=\mathrm{K}_{2}$, $\mathrm{S}=\mathrm{C}_{\mathrm{m} 2} / \mathrm{C}_{\mathrm{m} 1}$ and $\mathrm{cmc}=\left(\mathrm{C}_{\mathrm{m} 1}+\mathrm{C}_{\mathrm{m} 2}\right) /\left(\left(\mathrm{C}_{\mathrm{m} 1} \mathrm{v}_{1}+\mathrm{C}_{\mathrm{m} 2} \mathrm{v}_{2}\right) \mathrm{K}_{\mathrm{m}}^{\mathrm{o}} \mathrm{K}_{\mathrm{m}}\right)$. These formulas conform to those reported (ref.11) for the non ideal behaviour of the cmc of a mixture of two surfactants. The $\mathrm{cmc}$ of a mixture of more than two surfactants can be easily derived.

\section{SOLUTES SUBJECT TO CHEMICAL EQUILIBRIUM}

When solutes undergo chemical equilibrium, the actual total concentration of the species $\mathrm{C}_{\mathrm{i}}$ in eq.(9) is no more the analytical concentration and the treatment so far shown must be modified. Yet, colloidal systems in presence of solutes that are weak acids or complexing agents are of paramount importance. They are usually called "functionalized": a lot of advantages over conventional surfactants and promising utilisations in a variety of fields were pointed out (ref.28).

From a basic point of view in addition to factors discussed, the charge of the colloid depends on parameters that can change every chemical equilibrium. Consider a species $Y$ ( a solute or a surfactant) which can undergo a series of chemical equilibria with a species $\mathrm{W}$ ( a ligand or hydrogen ions):

$$
\begin{aligned}
& \mathrm{Y}+\mathrm{W}<==>\mathrm{YW} \\
& \mathrm{YW}+\mathrm{W}<==>\mathrm{YW}_{2} \\
& \cdots \cdots \ldots \ldots \ldots \ldots \ldots \ldots \ldots \ldots \ldots \ldots \ldots \ldots \ldots \ldots \ldots \\
& \mathrm{YW}_{\mathrm{n} \cdot 1}+\mathrm{W}<==>\mathrm{YW}_{\mathrm{n}}
\end{aligned}
$$

where each equilibrium is described by an equilibrium constant $K_{i}^{*}(i=1, n)$ in the absence of colloids. In the presence of a colloidal system and considering the pseudophase 
approximation, the partition of each species of reactions (12) is regulated by eq.(9). The fraction, over the total analytical concentration of $\mathrm{Y}\left(\mathrm{Cy}_{0}\right)$, of the equilibrium total concentration of the chemical species $\mathrm{YW}_{\mathrm{i}}\left(\mathrm{C}_{\mathrm{i}}\right.$, to be used in eq.9) is then given by:

$$
\frac{C_{i}}{C_{0}^{y}}=\frac{\beta_{i}^{a p p}\left[W_{f}\right]^{i}\left(1+K_{w}\right)^{i}}{1+\sum_{j=1}^{n} \beta_{j}^{a p p}\left[W_{f}^{j}\left(1+K_{w}\right)^{j}\right.}
$$

where $\beta_{i}$ app is the apparent (or conditional) equilibrium constant for the species $\mathrm{YW}_{\mathrm{j}}$ at a given surfactant concentration,

$$
\beta_{i}^{\text {app }}=\prod_{j=1}^{i} K_{j}^{*} \frac{\left(1+K_{j}\right)}{\left(1+K_{j-1}\right)\left(1+K_{w}\right)} \quad \text { and } \quad K_{k}=K^{{ }_{0}} K^{e_{k}} \frac{\varphi}{(1-\varphi)}
$$

In the case of $\mathrm{pH}$ measurements, $\left[\mathrm{W}_{\mathrm{f}}\right] \Rightarrow\left[\mathrm{H}^{+}\right]$, and $\left[\mathrm{H}^{+}\right]$can be measured. When the free concentration $\left[W_{f}\right]$ is not known, the quantity $C_{w}=\left[W_{f}\right]\left(1+K_{w}\right)$ can be calculated from the analytical concentration $\mathrm{C}_{\mathrm{o}}$ by solving for $\mathrm{C}_{\mathrm{w}}$ the polynomial:

$$
\frac{C_{w_{0}}-C_{w}}{C_{0} C_{w}}=\frac{\sum_{j=1}^{n} j \beta_{j}^{a p p} C_{w}^{(j-1)}}{1+\sum_{j=1}^{n} \beta_{j}^{a p p} C_{w}^{j}}
$$

Other more complex relationships are easily derived in the case of multiple crossed equilibria, for example when complexing and acid-base properties are present on the same ligand. Numerous algorithms reported in literature (ref. 29) handle the numerical solutions of such equations. All of them apply to the present model by simply replacing the formation constant with the expression given in eq. (13b).

When system (12) refers to the surfactant itself, i.e. the surfactant has multiple chelation sites or possesses multiple dissociable acid sites, the cmc is given by eq.(15) (assuming that all the $\mathrm{YW}_{\mathrm{i}}$ species have the same molar volume $\mathrm{v}_{\mathrm{m}}$ ) and the concentration of micellized surfactant $\left(C_{d}=\Sigma\left[Y W_{i}\right]_{\text {bound }}\right)$ is simply given by $C_{d}=C_{m}-c m c$.

$$
\begin{aligned}
& 1+\sum_{j=1}^{n} \beta_{j}\left[W_{f}\right]^{j} \\
& \mathrm{cmc}= \\
& v_{m}\left(K_{0}^{\circ} K^{e_{0}}+\sum_{j=1}^{n} \beta_{j} K^{\circ} K_{j}{ }_{j}\left[W_{f}{ }^{j}\right)\right.
\end{aligned}
$$

where $\beta_{j}=\prod K_{i}^{*}$ is the formation constant in water for the species $Y W_{j}$.

The use of eqs.(13)-(15) for the solution of the system $(5,7)-(9)$ increases the numerical difficulties but does not change the algorithm used in the simplest cases. These equations completely define the colloidal system even when complex chemical equilibria are present, provided that a hydrophobic transfer constant is assigned to each species and the equilibrium constants in water are known.

Eq.(15) reduces to eq.(10) when the surfactant does not participate to equilibria and shows that the reciprocal cmc is a linear combination of the transfer constants of the monomer weighted by the actual concentration fractions in the bulk solution, calculated neglecting the presence of a second pseudophase. For example, the cmc of a carboxylic acid 
surfactant (i.e. an alkylpolyoxyethylenecarboxylic acid) is cmc $=\left(\mathrm{K}_{\mathrm{a}}+[\mathrm{H}]^{+}\right) /$ $\left(\left[\mathrm{H}^{+}\right] \mathrm{K}^{\circ}{ }_{\mathrm{HA}}+\mathrm{K}_{\mathrm{a}} \mathrm{K}_{\mathrm{A}}^{\circ} \mathrm{K}_{\mathrm{A}}\right)$ where $\mathrm{K}_{\mathrm{a}}$ is the acid dissociation constant in water and transfer constants refer to the species HA and A. For a monoprotic acid the behaviour of cme versus $\mathrm{pH}$ is reported in Fig.3. It is evident that cmc corresponds to individual cmc of the two species in the acid and basic $\mathrm{pH}$ regions. The difference between $\mathrm{cmc}_{\mathrm{HA}}$ and $\mathrm{cmc}_{\mathrm{A}}$ is due to $\mathrm{K}^{\circ}{ }_{\mathrm{A}}$, which in turn depends on the concentration of added salt. The transition region obviously depends on the $\mathrm{pK}_{\mathrm{a}}$ of the acid (Fig.3, right).
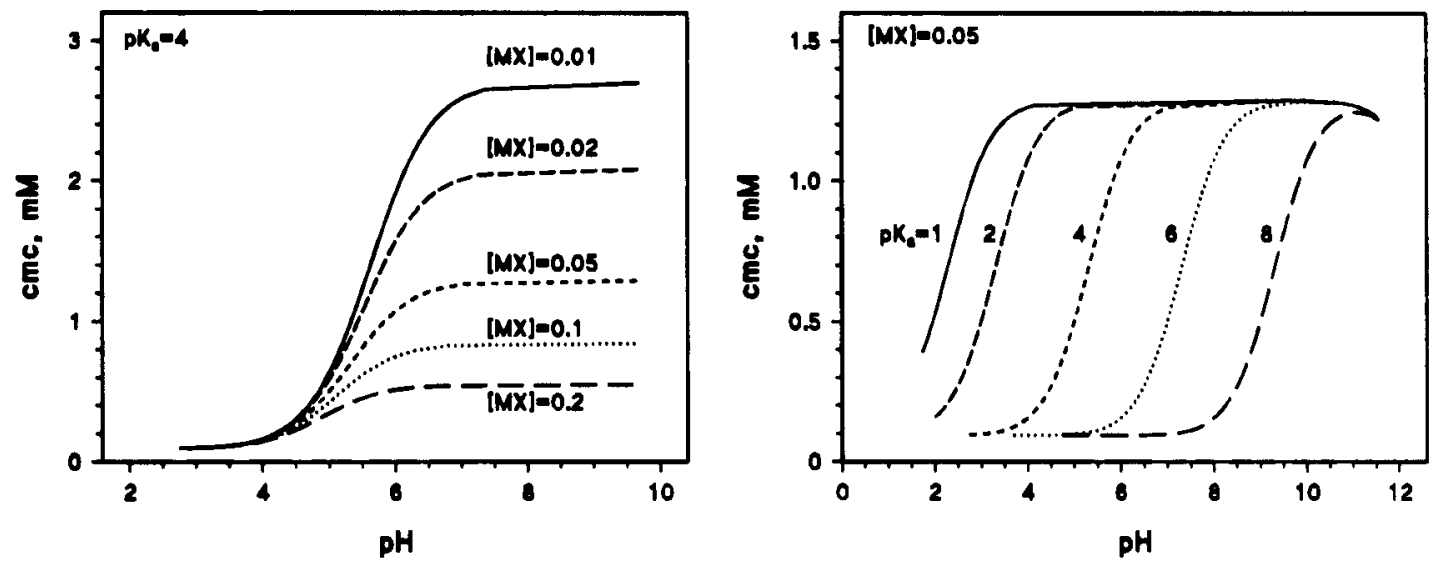

Fig.3. Calculated values of concentration of free surfactant according to eq.(15) for a weak acid surfactant $\mathrm{SAH} \Leftrightarrow \mathrm{SA}^{-}+\mathrm{H}^{+}$as a function of $\mathrm{pH}$ at different concentrations of excess inert salt $\mathrm{MX}$ (left) and with different values of $\mathrm{pK}_{\mathrm{a}}$ (right). Radii of all ions $2.5 \AA, \mathrm{K}^{\circ} \mathrm{HA}=\mathrm{K}^{\circ} \mathrm{A}=20000, \mathrm{~K}^{\circ}{ }_{\mathrm{M}}=\mathrm{K}^{\circ}{ }_{\mathrm{X}}=5, \mathrm{~K}^{\circ} \mathrm{H}^{+}=\mathrm{K}^{\circ} \mathrm{OH}^{-}=1$, micelle radius $25 \AA$. Variation of $\mathrm{pH}$ is calculated assuming a titration with $\mathrm{MOH}$ of $0.05 \mathrm{M}$ of surfactant.

The presence of colloids have also relevant effects on the apparent equilibrium constants (see eq.13b). The pK shifts observed in acid-base systems were already discussed in a critical review (ref.16) and the application of the formalism here presented to acid-base and complexometric titrations in the presence of micelles and other colloidal systems is in development.

\section{KINETIC EXPERIMENTS}

The kinetics of a reaction $A+B \rightarrow C+D$ can be expressed in the pseudophase model as:

$$
\frac{k_{o b s}}{k_{w}}=\frac{1-v_{m} C_{d}+k_{m i c} k_{w} K^{o}{ }_{A} K^{\bullet}{ }_{A} K^{\circ}{ }_{B} K^{\bullet}{ }_{B} v_{m} C_{d}}{\left(1+v_{m} C_{d}\left(K^{\circ}{ }_{A} K^{e}{ }_{A}-1\right)\left(1+v_{m} C_{d}\left(K^{\circ}{ }_{B} K^{\bullet}{ }_{B}-1\right)\right.\right.}
$$

Simple formulas for the kinetics as well as for the other phenomena related to the surfactant aggregation can be derived by using explicit and definite approximations. The possibility of treating the monomer-micelle equilibria, of calculating the micellar composition and $C_{d}$ in the frame of the same theoretical approach used to analyse the kinetic data, assures consistency in the treatment and permits a confident evaluation of the micellar charge. Application of the model to electron transfer kinetics in mixed micellar systems and in the presence of different counterions was promising (ref.13,14). 


\section{Acknowledgements}

Authors are grateful to CNR (Progetto Finalizzato) and to ENIRICERCHE for financial support.

\section{REFERENCES}

1. C. Tanford, The Hydrophobic Effect, Wiley, New York, 1973.

2. J. N. Israelachvili, D. J. Mitchell and B.N. Ninham, J.Chem.Faraday Trans.2 72:1525 (1976)

3. R. F. Kamrath and E. I. Frances, J.Phys.Chem 88, 1642 (1984)

4. J. Desnoyers, G. Perron and A. Roux, in Surfactants Solutions: New Methods of Investigation, R. Zana ed., ch 1, Surf. Ser. 22, Dekker, N.Y. (1987) and references cited

5. B. Jönsonn and H. Wennerström, J.Phys.Chem. 91, 338 (1987).

6. R. Nagarajan and E. Ruckenstein, J.Colloid Interface Sci. 60, 221 (1977); R. Nagarajan, Langmuir 1, 331 (1985)

7. J. Maeda, J.Phys.Chem. 92, 4490 (1988)

8. M. R. Böhmer, L. K. Koopal and J. Lyklema, J.Phvs.Chem. 95, 9569 (1991)

9. K. Shinoda, J. Phys. Chem. 58, 541 (1954)

10. Y. Moroi, N.Nishikido and R.Matuura, J.Colloid Interface Sci. 50, 344 (1975)

11. P. M. Holland and D. N. Rubingh, J.Phys.Chem. 87, 1984 (1983).

12. J. F. Rathman and J.F. Scamehorn, J.Phys.Chem 88, 5807 (1984)

13. C. Minero, E. Pramauro and E. Pelizzetti, J.Phys.Chem. 92, 4670 (1988)

14. C. Minero, A. Sassi and E.Pelizzetti, presented at ECIS Conference, Copanello, Italy (1990); in preparation

15. C. Minero and E. Pelizzetti, in Organized Solutions, S.E.Friberg and B.Lindman eds., ch 21 , Surf. Ser. 44, Dekker, N.Y. (1992)

16. C. Minero and E. Pelizzetti, Adv.Coll.Interface Sci. 37, 319 (1992)

17. A. Ben-Naim, Hydrophobic Interactions, Plenum Press, N.Y. (1980)

18. H. Wennerström, in Organized Solutions, S.E.Friberg and B.Lindman eds., ch 1, Surf. Ser. 44, Dekker, N.Y. (1992)

19. see for references: R. Zana, Cationic Surfactants, D.N. Rubingh and P.M. Holland eds., ch 2, Surf. Ser. 37, Dekker, N.Y. (1991)

20. B. Smith, P. A. J. Hilbers, K. Esselink, L. A. M. Rupert and N. M. van Os, Nature 348 , 264 (1990)

21. J. B. Hayter anf J. Penfold, J.Colloid Polvm.Sci. 261, 1022 (1983)

22. L. Benjamin, J. Phys. Chem. 68, 3575 (1964)

23. E. Rodenas, C.Dolcet and M.Valiente, J. Phys. Chem. 94, 1472 (1990)

24. H. Oshima, T. W. Healy and L.E. White, J.Colloid Interface Sci. 90, 17 (1982)

25. P. Mukerjee, Adv. Colloid Interface Sci. 1, 241 (1967)

26. C. Minero, E.Pramauro, E.Pelizzetti, V. Degiorgio and M.Corti, J. Phys. Chem. 90, $1620,(1986)$

27. G. Porte and J. Appell, J. Phys. Chem 85, 2511 (1981)

28. E. Pramauro, E.Pelizzetti, C.Minero, E.Barni, P.Savarino and G.Viscardi, Ann. Chim. (Rome) 77, 209 (1987)

29. A. De Robertis, C. De Stefano and S. Sammartano, Anal Chim. Acta 191, 385 (1986) 